\title{
CONSIDERAÇÕES TAXONÔMICAS NO GÊNERO STRYPHNODENDRON MART. (LEGUMINOSAE-MIMOSOIDEAE) E DISTRIBUIÇÃO GEOGRÁFICA DAS ESPÉCIES
}

\author{
Elena Maria de Lamare Occhioni'
}

\begin{abstract}
RESUMO - No presente trabalho são tratadas as modificações taxonômicas ocorridas no gênero Stryphnodendron Mart. ao longo de sua revisão nomenclatural, sendo também caracterizado por grupos, de acordo com a fitogeografia e o hábito de seus taxa.
\end{abstract}

Palavras-chave: Stryphnodendron., Taxonomia, Fitogeografia.

ABSTRACT - Recent taxonomic changes in the genus Stryphnodendron Mart. are reported. The genus is also characterized according to the phytogeography and the habit of its taxa.

Key words: Stryphnodendron, Taxonomy, Phytogeography.

\section{Introdução}

O gênero Stryphnodendron é tipicamente brasileiro, uma vez que aqui ocorrem 94\% dos taxa que o compõem. Apenas S. levelii Cowan (1958) tem sua ocorrência registrada somente para a Venezuela, e $S$. porcatum Neill \& Occh. f. (1989), até o momento, só foi encontrado no Equador.

Em decorrência de numerosas consultas relacionadas ao atual status de algumas espécies muito conhecidas, tornou-se necessária a divulgação das modificações taxonômicas do grupo, que se referem a espécies encontradas em diferentes formações fitogeográficas. Exemplo significativo é $S$. adstringens (Mart.) Coville, de ocorrência nos campos e cerrados brasileiros, que teve sua nomenclatura confusa durante muito tempo, e ainda hoje é tratada freqüentemente por diversos binômios, tais como $S$. barbatimão Mart., $S$. barbadetiman (Vell.) Mart., bem como exemplares de S. microstachyum Poepp. \& Endl., muito conhecidos na região amazônica ainda como $S$. purpureum Ducke.

1 - Professora do Departamento de Botânica do Instituto de Biologia da Universidade Federal do Rio de Janeiro - CCS Bloco A CEP 21941 - Cidade Universitária, Ilha do Fundão, Rio de Janeiro - RJ. 


\section{Resultados}

\section{Distribuição Geográfica do Gênero '}

O gênero Stryphnodendron apresenta sua área de dispersão limitada ao continente sulamericano, com centro provavelmente na Amazônia, onde ocorre a maior concentração de espécies (Figura 1).

Nota-se neste grupo fatos de ordem fitogeográfica e ecológica de grande importância, além dos de natureza morfológica, pois são encontradas no gênero desde espécies anãs, prostadas, características dos cerrados do Brasil Central, até as grandes árvores (cerca de $35-40$ metros de altura) como as que ocorrem na floresta amazônica.

O gênero compreende atualmente 32 taxa, sendo 29 espécies, 1 subespécie e 2 variedades, subdivididos em 2 seções, com distribuição geográfica e hábito bastante característicos, o que permite separá-los em grupos:

1) 14 taxa ocorrem na floresta Amazônica, sendo todos árvores, em sua maioria de grande porte, atingindo em média $35 \mathrm{~m}$ de altura:

S. occhianum Martins

S. paniculatum Poepp \& Endl.

$S$. polystachyum (Miq.) Kleinh.

$S$. racemiferum (Ducke) W. Rodr.

S. microstachyum Poepp. \& Endl.

S. levelii Cowan

S. foreroi Martins

S. rizzinianum Martins

S. pulcherrimum (Willd.) Hochr.

$S$. guianense (Aublet) Benth Subsp. guianense var. guianense

$S$. guianense (Aublet) Benth. subsp. guianense var. roseiflorum Ducke

$S$. guianense (Aublet) Benth. subsp. glandulosum Forero

S. duckeanum Occh.

$S$. porcatum Neill \& Occh. f.

2) 1 taxon arbóreo, cerca de 20 metros de altura, em floresta semidecídua seca, região de transição para o cerrado, Mato Grosso:

$S$. fissuratum Martins

3) 1 taxon arbóreo, cerca de 10 metros de altura, de ocorrência característica em mata pluvial, Pernambuco:

$S$. consimile Martins

4) 4 taxa arbustivos, com altura entre 4-7 metros, dos campos e cerrados do Brasil Central, Minas Gerais e São Paulo:

$S$. rotundifolium Mart.

$S$. obovatum Benth.

$S$. goyazense Taub.

$S$. adstringens (Mart.) Coville 
5) 2 taxa arbustivos, de ocorrência típica nas caatingas do nordeste brasileiro:

$S$. coriaceum Benth.

S. piptadenioides Martins

6) 2 taxa arbóreos de ocorrência típica nas matas da Serra da Mantiqueira, Estado do Rio de Janeiro:

$S$. polyphyllum Mart. var. polyphyllum

S.polyphyllum Mart. var. villosum Benth.

7) 8 taxa subarbustivos, denominados "plantas anãs", com no máximo $0,50 \mathrm{~cm}$ de altura, característicos dos cerrados de Goiás e Minas Gerais:

$S$. humile Martins

$S$. confertum Her. et Rizz.

$S$. plastyspicum Rizz. et Her.

$S$. gracile Her. et Rizz.

$S$. cristalinae Her.

$S$. heringeri Occh. f.

$S$. barbatulum Rizz. et Her.

$S$. sallesianum Her. et Rizz.

Classificação quanto ao hábito:

Baseado nas formas de vida dos taxa, Rizzini e Heringer (1987) dividiram o gênero Stryphnodendron em duas seções distintas:

Seção Stryphnodendron, englobando todos os taxa arbustivos e arbóreos dotados de caule simples.

Seção Elenaea Rizz. et Her., compreendendo 8 taxa de porte subarbustivo, por vezes prostrados, conhecidos como "plantas anãs", dotados de caule muito ramificado, de ocorrência limitada às regióes de queimadas periódicas, que destroem suas partes aéreas, e que apresentam brotação anual devido à resistência de sua parte caulinar subterrânea: $S$. confertum, $S$. platyspicum, $S$. humile, $S$. cristalinae, $S$. gracile, $S$. barbatulum, $S$. sallesianum e $S$. heringeri.

Alterações na nomenclatura de alguns taxa já propostos anteriormente:

1. S. adstringens (Mart.) Coville, Cent. Dict. et Cycl. 11:111. 1910 Sua nomenclatura esteve bastante confusa muitos anos, tendo sido corrigida por Forero (1972). Occhioni Martins (1973) fez comentário sobre a nomenclatura desta espécie, porém baseada em dados duvidosos sobre a verdadeira data de publicação da Flora Fluminense de Velloso. Numerosas exsicatas apresentam-se com determinações variadas, tais como $S$. barbatimão, $S$. barbatiman, 
$S$. barbadetiman além de citaçōes incorretas em diversos trabalhos consultados. Assim temos como sinonímia:

- Ac:acia adstringens Martius, Reise Bras. 2:548. 1828.

- Mimosa barbadetiman Velloso, Atlas 11, tab. 7. 1835.

- S. barbatimão Martius, Herb. Fl. Br. 117; Fl. Bras. 15(2): 284.1876.

- $S$. barbatimam Martius, Flora 20. 2:117. 1837.

- Mimosa virginalis Koster, Voyages Part. Sept. Brésil. 508. 1818 (nom. nud.).

2. S. microstachyum Poepp. et Endl., Nov. Gen. Sp. 3: 82. $1845-$ Baseada em estudo comparativo de numerosas exsicatas determinadas como $S$. purpureum e $S$. excelsum, Occhioni Martins (1979) verificou a ausência de caracteres morfológicos marcantes que justificassem a manutenção das mesmas como entidades independentes, motivo pelo qual as colocou na sinonímia de S. microstachyum:

- S. inaequale Benth., Trans. Linn. Soc. 30(3): 374. 1875.

- $S$. pupureum Ducke, Arch. Jard. Bot. R. Jan. 1:16. 1915.

- S. excelsum Harms, Fedde Repert. 19:646. 1923.

3. S. obovatum Benth. Trans. Linn. Soc. 30(3): 374.'1875. - Occhioni Martins (1979) também considerou como sinonímia a forma retusa Chodat e Hassler, baseada no caráter "retuso" que verificou ocorrer em várias gradações em todo o material examinado da espécie:

- $S$. rotundifolium Benth. f. retusa Chodat et Hassler, Bull. Herb. Boiss. Ser. 2.4:559. 1904.

4. S. pulcherrimum (Willd.) Hochr., Bull. N.Y. Bot. Gard. 6:274. 1910 Foram também colocadas na sinonímia desta espécie, por Occhioni Martins (1979), espécies muito conhecidas na região da floresta amazônica como $S$. angustum Benth., $S$. melinonis Sagot. e $S$. guianense (Aublet) Benth. var. floribundum Ducke. Considerou insuficientes as diferenças morfológicas observadas, para mantê-las como entidades independentes:

- Acacia pulcherrima Willd. Sp. Pl. 4:1061. 1806.

- Mimosa pulcherrima Poiret, in Lam. Encycl. Dict. Suppl. 1:66. 1810.

- Stryphnodendron floribundum Benth., Hook Journ. Bot. 4:343. 1842.

- $S$. angustum Benth., Trans. Linn. Soc. 30(3): 375. 1875.

- $S$. melinonis Sagot, Ann. Soc. Nat. Ser. 6(13): 322. 1882.

- $S$. guianense (Aublet) Benth. var. floribundum Ducke, Arch. Jard. Bot. R. jan. 4:250. 1925.

5. S. guianense (Aublet) Benth. subsp. guianense var. roseiflorum Ducke, Arch. Jard. Bot. R. Jan. 6:15. 1933 -Ducke (1933) descreveu S. guianense var. roseiflorum, elevada posteriormente à categoria de espécie pelo próprio autor (1944) e mais tarde restabelecida a sua condição de variedade por Occhioni Martins e Guedes Martins (1972). No exame de numerosas exsicatas determinadas como $S$. roseiflorum, estes autores verificaram ser impossível a 


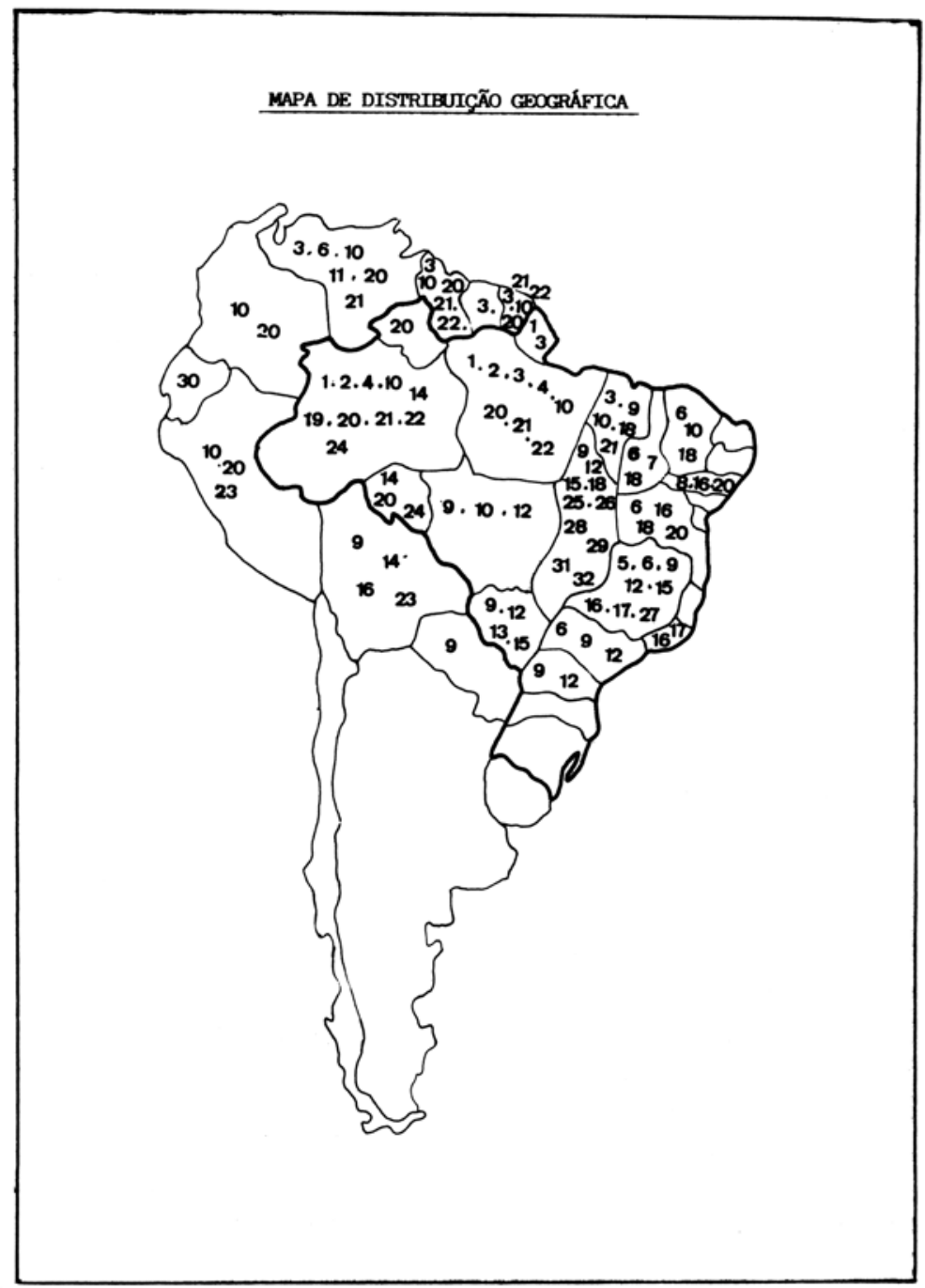

Figura 1 - Distribuição geográfica. 1. S. occhionianum; 2. S. paniculatum; 3. S. polystachyum; 4. S. racemiferum; 5. S. humile; 6. S. rotundifolium; 7. S. piptadenioides; 8. S. consimile; 9. S. obovatum; 10. S. microstachyum; 11. S. levelii; 12. S. adstringens; 13. S. fissuratum; 14. S. foreroi; 15. S. goyazense; 16. S. polyphyllum var. polyphyllum; 17. S. polyphyllum var. villosum; 18 . S. coriaceum; 19 . S. rizzinianum; 20. S. pulcherrimum; 21. S. guianense subsp. guianense var. guianense; 22. S. guianense subsp. guianense var. roseiflorum; 23. S. guianense subsp. glandulosum; 24. S. duckeanum; 25. S. confertum; 26. S. plastyspicum; 27. S. gracile; 28. S. cristalinae; 29. S. heringeri; 30. S. porc tum; 31. S. barbatulum; 32. S. sallesianum. 
observação dos caracteres em que Ducke (1944) se baseou para elevá-la à categoria de espécie, entre eles a forma das espigas levemente encurvadas "ao vivo".

- S. roseiflorum Ducke, Bol. Tec. Inst. Agron. Norte 2:8. 1944.

Limites de distribuição geográfica

O gênero Stryphnodendron se caracteriza como neotropical, apresentando como limite norte a Costa Rica, na América Central, onde foi registrada a ocorrência de uma única espécie e, como limite sul, o Estado do Paraná, no Brasil, com o registro de duas espécies.

Dos 32 taxa do gênero, 30 ocorrem em nosso território. Na Venezuela ocorrem apenas 6; nas Guianas 5; 4 na Bolívia; 3 no Peru; na Colômbia 2 e apenas 1 no Paraguai, Costa Rica, Suriname e Equador.

Foi possível verificar também que 18 taxa só têm ocorrência registrada, até o momento, para o Brasil: $S$. occhionianum, $S$. paniculatum, $S$. racemiferum, $S$. humile, $S$. piptadenioides, $S$. consimile, $S$. adstringens, $S$. fissuratum, $S$. goyazense, $S$. coriaceum, $S$. rizzinanum, $S$. polyphyllum, var. villosum, S.duckeanum, $S$. confertum, $S$. platyspicum, $S$. gracile, $S$. cristalinae, $S$. heringeri.

\section{Referências Bibliográficas}

COWAN, R.S. 1958. The botany of the Guayana Highland 3. Mém. New York Bot. Gard. 1O(1): 144-5. USA.

DUCKE, A. 1933. Plantes nouvelle ou peu connues de la région amazonienne IV. Arq. Jard. Bot. R. Janeiro. 6:15.

DUCKE, A. 1944. New or noteworthy Leguminosae on the Brasilian Amazonian. Bol. Técn. Inst. Agron. Norte. 2:8-9.

FORERO, E. 1972. Studies in Stryphnodendron (Leguminosae-Mimosoideae) including two new taxa. Brittonia, 24: 143-147.

NEILL, D.A. \& E.M. DE L. OCCHIONI. 1989. A new species of Stryphnodendron (Fabaceae: Mimosoieae) from Amazonian Ecuador. Ann. Missouri Bot. Gard. 76: 357-359.

OCCHIONI MARTINS, E.M. \& A. GUEDES MARTINS. 1972. STRYPHNODENDRON Mart. (Leg. Mim.) as espécies da amazônia brasileira I. Leandra 2(2): 11-40.

OCCHIONI MARTINS, E.M. 1973. Sobre a nomenclatura científica do barbatimão do Brasil. Leandra 2(2): 121-123.

OCCHIONI MARTINS, E.M. 1979. Sobre a sinonímia científica de espécies de Siryphnodendron Mart. (Leg. Mim.). Leandra 8-9(8-9): 85-89.

RIZZINI, C.T. \& E.P. HERINGER 1987. As espécies anãs de Stryphnodendron Mart. (Leguminosae - Mimosoideae). Rev. Bras. Biol. 47(3): 447-454. 